\title{
Long Haired Chihuahua
}

National Cancer Institute

\section{Source}

National Cancer Institute. Long Haired Chihuahua. NCI Thesaurus. Code C53930.

The Long Haired Chihuahua is a variety of chihuahua with a long-haired coat. 